\title{
Health-Related Quality of Life Among Rural Chinese Patients With Upper Gastrointestinal Diseases at Different Pathological Stages: A Cross-Sectional Study
}

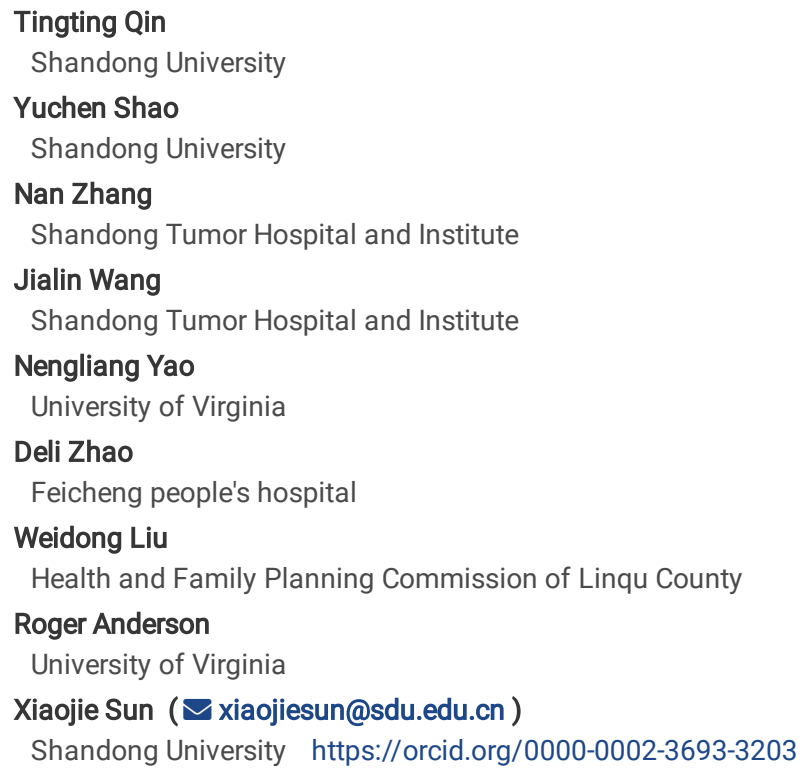

\section{Research}

Keywords: Upper gastrointestinal diseases, Esophageal cancer, Gastric cancer, Health-related quality of life, Pathological stage, Influencing factor

Posted Date: June 2nd, 2020

DOI: https://doi.org/10.21203/rs.3.rs-31481/v1

License: (c) (1) This work is licensed under a Creative Commons Attribution 4.0 International License. Read Full License 


\section{Abstract}

Objective: The aim of this study was to compare the health-related quality of life (HR-QoL) among rural Chinese patients with upper gastrointestinal (GI) diseases at different pathological stages and to determine the factors influencing HR-QoL in this population.

Methods: A cross-sectional survey was conducted in two counties in Shandong province in China from July to September 2018. Demographic and clinical characteristics were collected, and HR-QoL of patients was assessed using a set of questionnaires, including the EORTC QLQ-C30 (V3.0), the QLQ-OES18 and the QLQ-STO22. Multivariate analysis of variance (MANOVA) and Kruskal-Wallis test were used to compare the HR-QoL of patients of different pathological stage subgroups, and multivariate regression analyses were used to determine influencing factors.

Results: A total of 406 patients were included in this study. The mean scores for patients with upper $\mathrm{GI}$ diseases were $74.88(S D=19.15), 8.04(S D=16.17)$ and $8.64(S D=16.96)$ for the global QoL scale of EORTC QLQ-C30, QLQ-OES18 and QLQ-STO22, respectively. There was no significant difference in most of the scales between the precancerous and early stage groups, but a significant difference in HR-QoL between the precancerous and advanced groups as well as between early and advanced groups. Residence area, age, education level and pathological stage were the significant factors influencing HR-QoL scores, controlling for other variables.

Conclusions: The overall HR-QoL of patients with advanced upper GI cancer needs to be managed as a component of cancer care. Measures should be taken to routinely identify patient symptoms so that follow-up care can alleviate patient symptom burden. Tailored treatment and care for patients with upper GI diseases of different age, educational level and pathological stage may be needed to help improve HR-QoL.

\section{Introduction}

Upper gastrointestinal (GI) diseases primarily include esophageal precancerous lesions or cancer and gastric cardia precancerous lesions or cancer. Esophageal cancer and gastric cancer are two major lethal malignancies in the world. Among the 18.1 million new cancer cases in the world in 2018 , esophageal cancer and gastric cancer accounted for $3.2 \%$ and $5.7 \%$ of the total, ranking seventh and fifth respectively, and they were respectively the sixth and third leading causes of deaths among all 9.6 million cancer deaths [1]. In China, the age-standardized incidence rate by world standard population (ASIRW) of esophageal cancer and gastric cancer was 13.9/100,000 and 20.7/100,000 respectively, and there were 283,433 esophageal-related deaths and 390,182 gastric-related deaths in 2018 [2]. These two cancers are common malignancies in China, which produce a heavy disease burden [3]. Although some measures have been taken to control these cancers, the incidence of esophageal and gastric cancer still, respectively, ranks sixth and third among all cancers in China [4]. Worse still, the prevalence of precancerous lesions of the upper GI tract is also very high in China, which means that many patients are at increased risk of developing upper GI cancer[5-7]. Thus, upper GI diseases remain a serious health threat in China.

Recent medical and public health advances have led to the better treatment of existing diseases, with the hope for cure remaining the most important goal for patients and providers. Most patients also value the ability to maintain an acceptable level of health-related quality of life (HR-QoL) during and after treatment. Existed evidence suggests that overall HR-QoL plays an important role in determining the treatment outcomes among patients with cancer [8]. Internationally, HR-QoL is now considered one of the main parameters to be evaluated in studies about GI cancer surgery results [9], and is valuable because these data can reflect the patient's subjective feelings, such as comfort or fatigue, which could facilitate better communication between doctors and patients and could aid in clinical decision making $[10,11]$. Therefore, it is crucial to have a better understanding of the HR-QoL of patients with upper GI diseases.

A number of papers have recently been published reporting data from HR-QoL assessment studies on patients with upper GI diseases. Most of these studies have examined the effects of different medicine or treatments on HR-QoL [12-16]; others have focused on the changes in HR-QoL in cancer survivors over time $[17,18]$. There have also been some studies assessing the HR-QoL of patients with precancerous lesions in esophageal or gastric cancer [19, 20]. In China, most of the studies on HR-QoL and GI cancer have examined the HR-QoL in patients at a specific stage or with a specific type of gastric cancer or esophageal cancer [21-23]. Few studies have explored HR-QoL in patients with GI diseases at different pathological stages. This study will provide a comprehensive view of the HR-QoL of patients with upper GI diseases by including patients at all stages in order to inform clinical decision-making

The aims of this cross-sectional study were to explore the differences in HR-QoL between patients with upper GI diseases at different pathological stages and examine the factors influencing their HR-QoL.

\section{Materials And Methods}

\section{Study design and data collection}

Based on the distribution of GI diseases in Shandong Province, China, a cross-sectional survey was conducted in Feicheng and Linqu, two counties with high prevalence rates of upper GI diseases, from July to September 2018. In each county, cluster sampling was used to extract the participants and, to ensure the representativeness of the sample, at least 20 cases of each subgroup of upper GI diseases were investigated. All participants were randomly selected from two clinical databases: the Upper Gastrointestinal Cancer Early Diagnosis and Early Treatment Registration Report of Feicheng (2014-2017) and the Early Diagnosis and Early Treatment Registration Report of Linqu (2014-2016). These two clinical databases are based on the Early Diagnosis and Early Treatment Program in Feicheng and Linqu counties funded by the central government, which screened high-risk population aged 40-69 years by upper gastrointestinal (GI) endoscopy in areas with high-prevalence of upper GI diseases.

Patients were categorized according to the WHO Classification of Tumors of the Digestive System (Fourth Edition) [25]. For the purpose of this study, participants who had been selected from the above databases were divided into six subgroups. For esophageal diseases, there were three subgroups: (1) the 
precancerous group (including mild esophageal hyperplasia, moderate dysplasia, severe dysplasia, and carcinoma in situ); (2) the early stage group (including esophageal intramucosal cancer and esophageal submucosal cancer); (3) the advanced stage group (esophageal invasive cancer). For gastric diseases, there were also three subgroups: (1) the precancerous group (including severe atrophic gastritis, severe intestinal metaplasia, low-grade intraepithelial neoplasia, and high-grade intraepithelial neoplasia); (2) the early stage group (including intramucosal cancer and submucosal cancer); (3) the advanced stage group (cardia and gastric invasive cancer). Each subgroup included at least 20 cases. The inclusion criteria were: patients were diagnosed with certain upper GI diseases by pathological examination before more than 6 months but less than 3 years to the survey date; age $\geq 40$ years old. The exclusion criteria were: patients had severe cognitive deficits or were unresponsive, or had other major diseases such as mental illness; patients who could not understand the content of the questionnaire. The face-to-face interviews were conducted by trained investigators to collect basic information and the HRQoL data. A total of 450 cases were randomly selected from two clinical databases. About $91 \%$ of cases participated in our survey. According to the exclusion criteria, 39 cases were excluded. Most of these excluded cases were those unable to answer the questionnaire due to death or serious illness, and other reasons including refusal to participate and unreachable. Finally, 406 (99\%) participants were included in the analysis after excluding incomplete questionnaires.

\section{HR-QoL evaluation}

The questionnaire consisted of two parts. The first part collected data about participant demographic and clinical characteristics. The second part was comprised of three Chinese-version HR-QoL instruments: one cancer-specific QoL instrument (the EORTC QLQ-C30 (V3.0)), one EORTC esophageal cancer module (the EORTC QLQ-OES18), and one EORTC gastric cancer module (the EORTC QLQ-STO22).

QLQ-C30 (V3.0), QLQ-OES18, and QLQ-STO22 were all developed by the European Organization for Research and Treatment of Cancer (EORTC). The validation of these three questionnaires has been proved by previously published studies by EuroQol Group members [26-28]. In China, these questionnaires have been translated into Chinese according to the EORTC guidelines and have been proved to be valid instruments in assessing the HR-QoL of Chinese patients [29-31]. The QLQ-C30 incorporates 30 items, which are divided into five functional scales (physical, role, cognitive, emotional, and social), three symptom scales (fatigue, pain, and nausea and vomiting), six single items, and a global health and quality-of-life (QoL) scale. The QLQ-OES18 has four symptom scales (dysphagia, eating restrictions, reflux and pain) and six single items (trouble swallowing saliva, choking when swallowing, dry mouth, trouble with taste, trouble with coughing, and speech difficulties). The QLQ-STO22 module contains 22 items, which are categorized into five symptom scales (dysphagia, eating restrictions, pain, reflux and anxiety) and four single items (dry mouth, taste, body image and hair loss). High scores on the function scales and global health and QoL scale indicate a better level of overall QoL while high scores on the symptom scale/item represent a worse QoL. Statistical analysis

Descriptive summary statistics were computed to obtain participant demographic and clinical characteristics. The HR-QoL scores for each scale were expressed as mean values (standard deviation (SD)). In order to identify the differences in various dimensions of HR-QoL among patients with upper GI diseases at different pathological stages, the scores were compared by using multivariate analysis of variance (MANOVA) or the Kruskal-Wallis test. Univariate analyses (using Student's t tests or Wilcoxon tests) as well as multivariate analyses (using linear regression models) were used to determine influencing factors. All statistical analyses were performed using SAS statistical software version 9.4 (SAS Institute Inc., Cary, NC). P-values < 0.05 were considered statistically significant.

\section{Results}

\section{Characteristics of patients}

Table 1 summarizes the characteristics of the patients. A total of 406 patients were included in this study, 183(45.1\%) from Feicheng and 223 (54.9\%) from Linqu. There were $290(71.4 \%)$ males and $116(28.6 \%)$ females, and the average age was 62 years $(S D=8)$. Among all of the patients, nearly two thirds of the patients (61.8\%) had been diagnosed with gastric cancer, and $38.2 \%$ of patients suffered from esophageal cancer. More than a half of them (62.3\%) were in the precancerous stage. 
Table 1

Demographic and clinical characteristics of participants

\begin{tabular}{|c|c|c|}
\hline Characteristics & All patients $(n=406)$ & Percentage (\%) \\
\hline \multicolumn{3}{|l|}{ Residence area } \\
\hline Feicheng & 183 & 45.1 \\
\hline Linqu & 223 & 54.9 \\
\hline \multicolumn{3}{|l|}{ Gender } \\
\hline Male & 290 & 71.4 \\
\hline Female & 116 & 28.6 \\
\hline \multicolumn{3}{|l|}{ Age } \\
\hline$\leq 62$ years & 204 & 50.2 \\
\hline$₫ 62$ years & 202 & 49.8 \\
\hline \multicolumn{3}{|l|}{ Educational level } \\
\hline Primary school and below & 171 & 42.1 \\
\hline Middle school and above & 235 & 57.9 \\
\hline \multicolumn{3}{|l|}{ Marital status } \\
\hline Married & 365 & 89.9 \\
\hline Unmarried & 41 & 10.1 \\
\hline \multicolumn{3}{|l|}{ Migrant worker ${ }^{a}$} \\
\hline Yes & 56 & 13.8 \\
\hline No & 350 & 86.2 \\
\hline \multicolumn{3}{|l|}{ Lesion location } \\
\hline Esophageal & 155 & 38.2 \\
\hline Gastric/cardia & 251 & 61.8 \\
\hline \multicolumn{3}{|l|}{ Pathological stage } \\
\hline Precancerous & 253 & 62.3 \\
\hline Early & 74 & 18.2 \\
\hline Advanced & 79 & 19.5 \\
\hline \multicolumn{3}{|l|}{ Family history of disease } \\
\hline Yes & 68 & 16.7 \\
\hline No & 338 & 83.3 \\
\hline
\end{tabular}

\section{HR-QoL scores of patients in different subgroups}

The EORTC QLQ-C30 scores are shown in Table 2. The mean score of global QoL was $74.88(S D=19.15)$. In general, people with advanced GI cancer had the lowest scores. Among the function scales, the social function mean scores ( 80.55 for esophageal diseases and 85.32 for gastric diseases) were the lowest. Among the three symptom scales, the mean scores for fatigue ( 23.10 for esophageal and 16.51 for gastric diseases) were the highest. The mean scores of financial difficulties (32.84 for esophageal diseases and 23.99 for gastric diseases) were the highest among six single items. There were no notable significant differences in HR-QoL between the precancerous and early stage groups. However, we observed significant differences in HR-QoL between the precancerous and advanced groups as well as between early and advanced groups. For patients with esophageal cancer, there was no significant difference in the scale of diarrhea. For patients with gastric cancer, we found significant differences in two function scales (physical and social) and four symptom scales (fatigue, appetite lose, diarrhea and financial difficulties) between the precancerous and early stage groups. 
Table 2

Mean scores (SD) of EORTC QLQ-C30 at different stage groups for patients with upper GI diseases

\begin{tabular}{|c|c|c|c|c|c|c|c|c|}
\hline \multirow[t]{2}{*}{ Scales/items } & \multicolumn{3}{|l|}{ Esophageal } & \multirow[t]{2}{*}{$p$ value } & \multicolumn{3}{|l|}{ Gastric } & \multirow[t]{2}{*}{$p$ value } \\
\hline & Precancerous & Early stage & Advanced & & Precancerous & Early stage & Advanced & \\
\hline Global QoL & $79.09(15.26)$ & $70.42(15.87)$ & ${ }_{c}^{56.25(30.62)}$ & $\begin{array}{l}< \\
0.0001^{\dagger}\end{array}$ & $79.46(14.94)$ & $77.47(17.78)$ & ${ }_{c}^{61.67(21.43)^{b}}$ & $\begin{array}{l}<.0001^{+} \\
0\end{array}$ \\
\hline \multicolumn{9}{|l|}{ Function scales } \\
\hline Physical & $92.68(11.87)$ & $88.00(10.94)$ & $\begin{array}{l}67.78(20.59)^{b} \\
c\end{array}$ & $\begin{array}{l}< \\
0.0001^{\ddagger}\end{array}$ & $95.73(9.90)$ & $\begin{array}{l}88.27(17.56) \\
\text { a }\end{array}$ & $82.55(16.95)^{b}$ & $\begin{array}{l}<.0001^{\dagger} \\
0\end{array}$ \\
\hline Role & $96.28(11.34)$ & $95.83(10.64)$ & $c_{c}^{68.75(27.94)^{b}}$ & $\begin{array}{l}< \\
0.0001^{\dagger}\end{array}$ & $97.77(7.75)$ & $92.28(17.65)$ & $\begin{array}{l}84.85(20.10)^{b} \\
c\end{array}$ & ${ }_{0.0001^{\dagger}}$ \\
\hline Cognitive & $89.14(14.27)$ & $87.50(14.18)$ & ${ }_{c}^{78.47(19.95)^{b}}$ & $0.0092^{\ddagger}$ & $94.48(10.64)$ & $93.83(14.22)$ & $\begin{array}{l}84.85(16.75)^{b} \\
c\end{array}$ & $\begin{array}{l}<.0001^{\dagger} \\
0 .\end{array}$ \\
\hline Emotional & $92.04(13.21)$ & $93.75(10.77)$ & $80.90(21.21)^{b}$ & $\begin{array}{l}<.0001^{\dagger} \\
0 .\end{array}$ & $94.25(10.84)$ & $90.90(14.86)$ & $\begin{array}{l}80.00(16.17)^{b} \\
c\end{array}$ & $\begin{array}{l}< \\
0.0001^{\ddagger}\end{array}$ \\
\hline Social & $92.26(15.49)$ & $85.50(21.56)$ & $\begin{array}{l}63.89(26.77)^{b} \\
c\end{array}$ & $\begin{array}{l}< \\
0.0001^{\dagger}\end{array}$ & $95.31(12.45)$ & $\begin{array}{l}86.11(21.41) \\
\text { a }\end{array}$ & ${ }_{c}^{74.55(22.87)^{b}}$ & ${ }_{0.0001^{\dagger}}^{<}$ \\
\hline \multicolumn{9}{|l|}{ Symptom scales/items } \\
\hline Fatigue & $11.51(15.28)$ & $16.11(17.47)$ & ${ }_{c}^{41.67(23.68)^{b}}$ & $\begin{array}{l}< \\
0.0001^{\ddagger}\end{array}$ & 7.82(13.42) & $\begin{array}{l}16.05(22.29) \\
\mathrm{a}\end{array}$ & ${ }_{c}^{25.66(18.99)}$ & $\begin{array}{l}<.0001^{\dagger} \\
0 .\end{array}$ \\
\hline $\begin{array}{l}\text { Nausea and } \\
\text { vomiting }\end{array}$ & $2.53(8.13)$ & $5.83(12.42)$ & $21.53(24.32)^{b}$ & $\begin{array}{l}< \\
0.0001^{\dagger}\end{array}$ & $2.93(8.26)$ & $4.63(11.86)$ & ${ }_{c}^{13.64(20.81)^{b}}$ & $\begin{array}{l}< \\
0.0001^{\dagger}\end{array}$ \\
\hline Pain & 7.44(13.94) & $9.17(11.44)$ & ${ }_{c}^{27.08(27.28)^{b}}$ & $\hat{0}_{0.0001^{\dagger}}$ & $5.63(13.30)$ & $10.80(17.48)$ & $c_{c}^{18.48(22.15)^{b}}$ & $\begin{array}{l}<.0001^{\ddagger} \\
0\end{array}$ \\
\hline Dyspnea & $4.76(12.54)$ & $6.67(13.68)$ & ${ }_{c}^{26.39(29.45)^{b}}$ & $\begin{array}{l}<.0001^{\dagger} \\
0 .\end{array}$ & $3.29(10.73)$ & $3.09(13.38)$ & ${ }_{c}^{15.15(22.05)^{b}}$ & $\begin{array}{l}< \\
0.0001^{\dagger}\end{array}$ \\
\hline Insomnia & 10.71(21.09) & $16.67(25.36)$ & ${ }_{c}^{31.94(30.26)^{b}}$ & $0.0004^{\ddagger}$ & $8.22(17.39)$ & $10.49(24.93)$ & ${ }_{c}^{16.97(22.10)^{b}}$ & $0.0262^{\dagger}$ \\
\hline Appetite loss & $7.14(15.77)$ & $8.33(18.34)$ & ${ }_{c}^{38.89(27.22)}$ & $\begin{array}{l}<.0001^{\dagger} \\
0 .\end{array}$ & $5.40(14.11)$ & $\begin{array}{l}11.73(24.36) \\
\text { a }\end{array}$ & $26.06(22.85)^{b}$ & $\begin{array}{l}<.0001^{\dagger} \\
0\end{array}$ \\
\hline Constipation & 7.44(17.16) & $6.67(17.44)$ & ${ }_{c}^{18.06(25.97)^{b}}$ & $0.0384^{\ddagger}$ & $2.11(9.90)$ & $6.17(20.55)$ & ${ }_{c}^{10.91(21.33)^{b}}$ & $0.0018^{\dagger}$ \\
\hline Diarrhea & $9.52(20.71)$ & $5.00(12.21)$ & $15.28(27.77)$ & $0.2664^{\ddagger}$ & $3.29(11.44)$ & $\begin{array}{l}10.49(24.08) \\
\mathrm{a}\end{array}$ & $12.73(23.56)^{b}$ & $0.0012^{\dagger}$ \\
\hline Financial difficulties & 15.18(26.05) & $41.67(32.22)$ & $\begin{array}{l}41.67(29.90)^{b} \\
c\end{array}$ & $\begin{array}{l}< \\
0.0001^{\ddagger}\end{array}$ & $9.62(22.66)$ & $\begin{array}{l}29.01(27.51) \\
\mathrm{a}\end{array}$ & $33.33(27.22)^{b}$ & $\begin{array}{l}< \\
0.0001^{\ddagger}\end{array}$ \\
\hline \multicolumn{9}{|c|}{ a Significant difference between the precancerous group and the early stage group. } \\
\hline \multicolumn{9}{|c|}{ b Significant difference between the precancerous group and the advanced stage group. } \\
\hline \multicolumn{9}{|c|}{ c Significant difference between the early stage group and advanced stage group. } \\
\hline \multicolumn{9}{|c|}{${ }^{\dagger}$ Kruskal-Wallis test was used. } \\
\hline
\end{tabular}

The EORTC QLQ-OES18 scores are shown in Table 3. The mean score of QLQ-OES18 was $8.04(S D=16.17)$. For patients with advanced esophageal cancer, the score was the highest. There were significant differences in two symptom scales (dysphagia and trouble with coughing) between the precancerous and early stage groups. In addition, significant differences in symptoms were observed between precancerous and advanced stage groups in most scales except for dry mouth and trouble with taste. We also found significant differences between early and advanced stage groups in seven symptom scales (dysphagia, eating difficulties, reflux, pain, trouble swallowing saliva and choked when swallowing, speech difficulties). Additionally, the reflux score was the highest among these three groups. 
Table 3

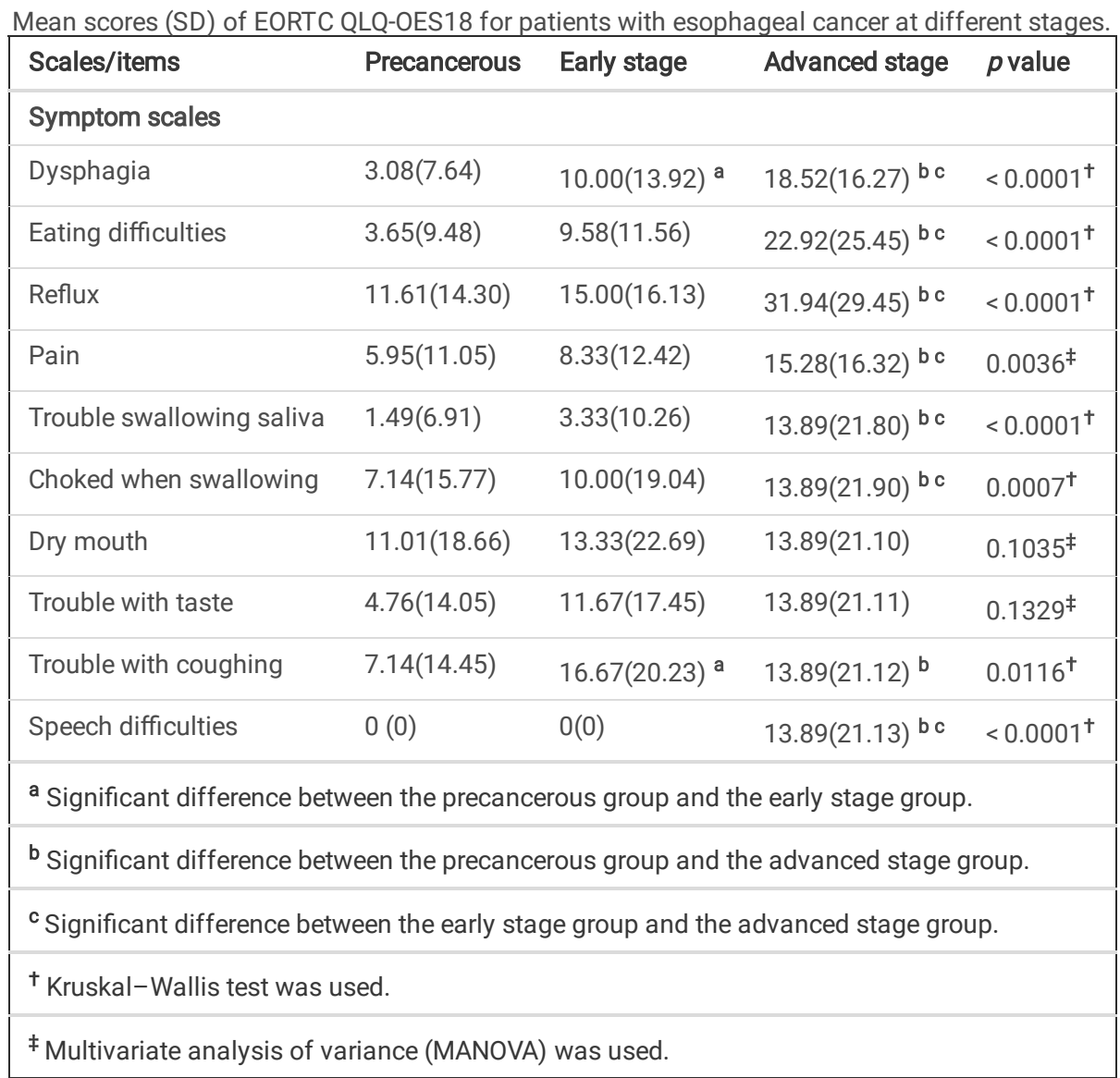

The EORTC QLQ-STO22 scores are shown in Table 4. The mean score of QLQ-STO22 was $8.64(S D=16.96)$. The advanced stage group had the highest score. Unlike esophageal cancer, among the gastric cancer patients, we found significant differences between precancerous and early stage groups in most symptom scales except for reflux, eating difficulties and hair loss. Significant differences in symptoms were observed between precancerous and advanced stage groups as well as between early and advanced groups in most scales except for body image, which had the highest scores of all the stage groups. 
Table 4

Mean scores (SD) of QLQ-ST022 for patients with gastric cancer at different stages.

\begin{tabular}{|c|c|c|c|c|}
\hline Scales/items & Precancerous & Early stage & Advanced stage & $p$ value \\
\hline \multicolumn{5}{|l|}{ Symptom scales } \\
\hline Dysphagia & $0.55(6.92)$ & $5.14(10.94)^{a}$ & $9.09(12.49)^{b c}$ & $<0.0001^{\dagger}$ \\
\hline Pain & $8.04(13.14)$ & $13.58(14.32)^{a}$ & $22.88(19.19) b c$ & $<0.0001^{\dagger}$ \\
\hline Reflux & $6.96(14.23)$ & 11.11(14.15) & $19.19(14.10) b c$ & $<0.0001^{\ddagger}$ \\
\hline Eating difficulties & $4.17(11.39)$ & $14.20(18.57)$ & $18.18(15.72)^{b c}$ & $<0.0001^{\dagger}$ \\
\hline Anxiety & 1.64(8.36) & $8.85(11.70)^{a}$ & $17.17(15.24)^{b c}$ & $<0.0001^{\dagger}$ \\
\hline Dry mouth & $1.17(10.05)$ & $11.11(21.48)^{a}$ & $20.00(22.77)^{b c}$ & $<0.0001^{\dagger}$ \\
\hline Taste & $1.41(11.14)$ & $6.79(17.59)^{a}$ & $15.76(19.09)^{b c}$ & $<0.0001^{\dagger}$ \\
\hline Body image & 11.03(22.36) & $28.40(23.71)^{a}$ & $30.91(24.72)^{b}$ & $<0.0001^{\dagger}$ \\
\hline Hair loss & $1.17(6.17)$ & $1.23(9.07)$ & $9.70(19.95) b c$ & $<0.0001^{\dagger}$ \\
\hline \multicolumn{5}{|c|}{ a Significant difference between the precancerous group and the early stage group. } \\
\hline \multicolumn{5}{|c|}{ b Significant difference between the precancerous group and the advanced stage group } \\
\hline \multicolumn{5}{|c|}{ ' Significant difference between the early stage group and the advanced stage group. } \\
\hline \multicolumn{5}{|c|}{${ }^{\dagger}$ Kruskal-Wallis test was used. } \\
\hline
\end{tabular}

\section{Factors associated with HR-QoL scores}

Factors influencing the scores of EORTC QLQ-C30, QLQ-OES18 and QLQ-STO22 in the univariate and multivariate analyses are shown in Tables 5-7.

As for EORTC QLQ-C30, we found that the pathological stage was an important factor influencing HR-QoL. Besides this factor, we found that age was a statistically significant factor affecting global QoL. Residence area and age were also significant influencing factors of physical functioning. Residence area, marital status and lesion location were major factors associated with cognitive functioning. Residence area was a significant factor in emotional functioning. Residence area and education level were two important factors affecting fatigue. Education level was a significant factor influencing appetite loss. Gender, education level, lesion location and family history of disease were significant factors affecting constipation. Residence area was a significant factor influencing both diarrhea and financial difficulties. 
Table 5

P-values for factors influencing EORTC QLQ-C30 scores in the univariate/multivariate analyses

\begin{tabular}{|c|c|c|c|c|c|c|c|c|c|}
\hline Scales/items & $\begin{array}{l}\text { Residence } \\
\text { area }\end{array}$ & Gender & Age & $\begin{array}{l}\text { Education } \\
\text { level }\end{array}$ & $\begin{array}{l}\text { Marital } \\
\text { status }\end{array}$ & $\begin{array}{l}\text { Migrant } \\
\text { worker }\end{array}$ & $\begin{array}{l}\text { Lesion } \\
\text { location }\end{array}$ & $\begin{array}{l}\text { Pathological } \\
\text { stage }\end{array}$ & $\begin{array}{l}\text { Family } \\
\text { history of } \\
\text { disease }\end{array}$ \\
\hline Global QoL & 0.731 & 0.509 & $0.003 / 0.001$ & $0.009 / 0.136$ & 0.181 & 0.338 & 0.833 & $\begin{array}{l}<.001 /<0.001 \\
0.00\end{array}$ & 0.676 \\
\hline \multicolumn{10}{|c|}{ Function scales } \\
\hline Physical & $0.006 / 0.001$ & 0.984 & $0.009 / 0.010$ & 0.068 & 0.061 & 0.835 & 0.058 & $\begin{array}{l}<.001 /<0.001 \\
0.00\end{array}$ & 0.288 \\
\hline Role & 0.292 & 0.375 & 0.232 & 0.838 & 0.830 & 0.815 & 0.308 & $<.001 /<0.001$ & 0.681 \\
\hline Cognitive & $\begin{array}{l}<.001 /<0.001 \\
\end{array}$ & 0.593 & 0.139 & 0.164 & $0.038 / 0.038$ & 0.782 & $0.001 / 0.001$ & $<0.001 /<0.001$ & 0.279 \\
\hline Emotional & $0.026 / 0.026$ & 0.565 & 0.460 & 0.875 & 0.255 & 0.258 & 0.911 & $\begin{array}{l}<.001 /<0.001 \\
0.00\end{array}$ & 0.638 \\
\hline Social & 0.365 & 0.218 & 0.155 & 0.166 & 0.250 & 0.172 & 0.390 & $\begin{array}{l}<.001 /<0.001 \\
\end{array}$ & 0.337 \\
\hline \multicolumn{10}{|c|}{ Symptom scales } \\
\hline Fatigue & $0.006 / 0.006$ & 0.892 & 0.103 & $0.020 / 0.021$ & 0.206 & 0.950 & 0.097 & $\begin{array}{l}<.001 /<0.001 \\
0.00\end{array}$ & 0.452 \\
\hline $\begin{array}{l}\text { Nausea and } \\
\text { vomiting }\end{array}$ & 0.678 & 0.593 & 0.101 & $0.003 / 0.001$ & 0.621 & 0.963 & 0.961 & $\begin{array}{l}<.001 /<0.001 \\
0.00\end{array}$ & 0.336 \\
\hline Pain & 0.170 & 0.678 & 0.298 & 0.224 & 0.508 & 0.431 & 0.598 & $\begin{array}{l}<.001 /<0.001 \\
0.00\end{array}$ & 0.750 \\
\hline Dyspnea & 0.263 & 0.951 & 0.125 & 0.058 & 0.347 & 0.897 & 0.301 & $\begin{array}{l}< \\
0.001 /<0.001\end{array}$ & 0.272 \\
\hline Insomnia & 0.120 & 0.136 & 0.639 & 0.339 & $0.012 / 0.051$ & 0.354 & 0.117 & $\begin{array}{l}<.001 /<0.001 \\
0.00\end{array}$ & 0.534 \\
\hline Appetite loss & 0.649 & 0.548 & 0.185 & $0.001 / 0.003$ & 0.662 & 0.410 & 0.649 & $\dot{0.001 /<0.001}$ & 0.322 \\
\hline Constipation & 0.225 & $0.007 / 0.003$ & 0.418 & $0.014 / 0.008$ & 0.480 & 0.467 & $0.008 / 0.022$ & $<.001 /<0.001$ & $0.024 / 0.002$ \\
\hline Diarrhea & $0.028 / 0.023$ & 0.616 & 0.098 & 0.589 & 0.954 & 0.926 & 0.101 & $0.007 /<0.001$ & 0.282 \\
\hline $\begin{array}{l}\text { Financial } \\
\text { difficulties }\end{array}$ & $0.004 / 0.003$ & 0.101 & 0.100 & 0.183 & 0.128 & 0.541 & 0.223 & $\begin{array}{l}<.001 /<0.001 \\
\end{array}$ & 0.307 \\
\hline \multicolumn{10}{|c|}{$\begin{array}{l}\text { Statistically significant } p \text {-values are underlined. Multivariate analyses were performed when multiple significant } p \text {-values were found in the univariate } \\
\text { analysis. }\end{array}$} \\
\hline \multicolumn{10}{|c|}{$\begin{array}{l}\text { Residence area: Feicheng vs. Linqu; gender: male vs. female; age: } \leq \text { vs. > } 62 \text { years; education level: primary school and below vs. middle school and } \\
\text { above; marital status: married vs. unmarried; migrant worker: yes vs. no; pathological stage: precancerous/early vs. advanced; family history of disease: } \\
\text { yes vs. no. }\end{array}$} \\
\hline
\end{tabular}


Table 6

P-values for factors influencing EORTC QLQ-OES18 scores in the univariate/multivariate analyses

\begin{tabular}{|c|c|c|c|c|c|c|c|c|}
\hline Scales/items & $\begin{array}{l}\text { Residence } \\
\text { area }\end{array}$ & Gender & Age & $\begin{array}{l}\text { Education } \\
\text { level }\end{array}$ & $\begin{array}{l}\text { Marital } \\
\text { status }\end{array}$ & $\begin{array}{l}\text { Migrant } \\
\text { worker }\end{array}$ & $\begin{array}{l}\text { Pathological } \\
\text { stage }\end{array}$ & $\begin{array}{l}\text { Family history of } \\
\text { disease }\end{array}$ \\
\hline \multicolumn{9}{|l|}{ Symptom scales } \\
\hline Dysphagia & 0.888 & 0.511 & 0.524 & 0.969 & 0,870 & 0.472 & $<0.001 /<0.001$ & 0.880 \\
\hline Eating difficulties & 0.972 & 0.640 & 0.110 & 0.527 & 0.077 & 0.610 & $<0.001 /<0.001$ & 0.340 \\
\hline Reflux & 0.298 & 0.354 & 0.730 & 0.533 & 0.436 & 0.551 & $0.001 /<0.001$ & 0.779 \\
\hline Pain & 0.594 & 0.453 & 0.370 & 0.369 & 0.706 & 0.456 & $0.009 / 0.004$ & 0.619 \\
\hline $\begin{array}{l}\text { Trouble swallowing } \\
\text { saliva }\end{array}$ & 0.427 & 0.130 & 0.512 & 0.993 & 0.515 & 0.427 & $<0.001 /<0.001$ & 0.734 \\
\hline $\begin{array}{l}\text { Choked when } \\
\text { swallowing }\end{array}$ & 0.386 & 0.553 & 0.968 & 0.928 & 0.801 & 0.196 & $0.001 /<0.001$ & 0.753 \\
\hline Dry mouth & 0.101 & 0.737 & 0.178 & 0.641 & 0.644 & 0.581 & 0.062 & 0.971 \\
\hline Trouble with taste & 0.864 & 0.591 & $0.046 / 0.128$ & 0.972 & 0.492 & 0.191 & 0.069 & 0.699 \\
\hline $\begin{array}{l}\text { Trouble with } \\
\text { coughing }\end{array}$ & 0.540 & 0.122 & 0.601 & 0.051 & 0.866 & 0.118 & 0.117 & 0.832 \\
\hline Speech difficulties & $0.018 / 0.018$ & 0.135 & 0.179 & 0.953 & 0.332 & 0.905 & $<0.001 /<0.001$ & 0.967 \\
\hline
\end{tabular}

Statistically significant $p$-values are underlined. Multivariate analyses were performed when multiple significant $p$-values were found in the univariate analysis.

Residence area: Feicheng vs. Linqu; gender: male vs. female; age: $\leq$ vs. $>62$ years; education level: primary school and below vs. middle school and above; marital status: married vs. unmarried; migrant worker: yes vs. no; pathological stage: precancerous/early vs. advanced; family history of disease: yes vs. no.

Regarding EORTC QLQ-STO22, we found that the pathological stage was a significant influencing factor in those symptom scales. In addition, residence area was a significant factor influencing reflux. Education level was a significant influencing factor of eating difficulties. Education level and martial status were two significant factors associated with dry mouth. Age and education level were major influencing factors of body image. Lastly, age was a significant influencing factor of hair loss.

Table 7

P-values for factors influencing EORTC QLQ-STO22 scores in the univariate/multivariate analyses

\begin{tabular}{|c|c|c|c|c|c|c|c|c|}
\hline Scales/items & $\begin{array}{l}\text { Residence } \\
\text { area }\end{array}$ & Gender & Age & $\begin{array}{l}\text { Education } \\
\text { level }\end{array}$ & $\begin{array}{l}\text { Marital } \\
\text { status }\end{array}$ & $\begin{array}{l}\text { Migrant } \\
\text { worker }\end{array}$ & $\begin{array}{l}\text { Pathological } \\
\text { stage }\end{array}$ & $\begin{array}{l}\text { Family history of } \\
\text { disease }\end{array}$ \\
\hline \multicolumn{9}{|c|}{ Symptom scales } \\
\hline Dysphagia & 0.829 & 0.330 & $0.042 / 0.261$ & $0.029 / 0.060$ & 0.435 & 0.530 & $<0.001 /<0.001$ & 0.262 \\
\hline Pain & 0.168 & 0.250 & 0.422 & $0.017 / 0.017$ & 0.944 & 0.978 & $<0.001 /<0.001$ & 0.844 \\
\hline Reflux & $0.007 / 0.011$ & 0.643 & 0.208 & 0.051 & 0.273 & 0.853 & $<0.001 /<0.001$ & 0.914 \\
\hline $\begin{array}{l}\text { Eating } \\
\text { difficulties }\end{array}$ & $0.022 / 0.101$ & 0.228 & 0.270 & $0.004 / 0.008$ & 0.182 & 0.522 & $<0.001 /<0.001$ & 0.448 \\
\hline Anxiety & 0.125 & 0.172 & $0.047 / 0.109$ & 0.160 & 0.673 & 0.830 & $<0.001 /<0.001$ & 0.604 \\
\hline Dry mouth & 0.632 & 0.534 & 0.064 & $0.028 / 0.017$ & $0.002 / 0.017$ & 0.870 & $<0.001 /<0.001$ & 0.421 \\
\hline Taste & 0.356 & 0.586 & 0.462 & 0.471 & 0.703 & 0.749 & $<0.001 /<0.001$ & 0.842 \\
\hline Body image & 0.183 & 0.498 & $0.045 / 0.045$ & $0.047 / 0.047$ & 0.451 & 0.454 & $<0.001 /<0.001$ & 0.979 \\
\hline Hair loss & 0.139 & 0.897 & $0.026 / 0.017$ & 0.159 & 0.691 & 0.862 & $<0.001 /<0.001$ & 0.802 \\
\hline
\end{tabular}

Statistically significant $p$-values are underlined. Multivariate analyses were performed when multiple significant $p$-values were found in the univariate analysis.

Residence area: Feicheng vs. Linqu; gender: male vs. female; age: $\leq$ vs. > 62 years; education level: primary school and below vs. middle school and above; marital status: married vs. unmarried; migrant worker: yes vs. no; pathological stage: precancerous/early vs. advanced; family history of disease: yes vs. no.

\section{Discussion}

HR-QoL has been widely accepted as an important health indicator by both healthcare providers and patients, functioning as a comprehensive evaluation of disease and treatment effects on patient wellbeing. In this cross-sectional study, we compared the HR-QoL of patients with upper GI diseases in different groups in rural China and examined the influencing factors of major HR-QoL dimensions. The results of this study can provide useful evidence regarding 
aspects of HR-QoL that are impaired due to upper GI diseases or their treatment among rural Chinese patients, and thus, may lead to better supportive treatment.

Our major finding was that there was no significant difference in HR-QoL between precancerous and early stage groups. In contrast, patients with advanced upper GI cancers showed worse HR-QoL in all function scales and most symptom scales, consistent with earlier studies [32, 33]. This finding suggests that doctors should pay more attention to deterioration in the HR-QoL of patients with advanced cancer, as well as seek to prevent deterioration of the relatively high levels of HR-QoL in early stage upper GI patients. Patients with upper GI diseases suffered from social problems, fatigue and financial difficulties, and may require appropriate social support to improve HR-QoL after treatment. As for gastric cancer, the results showed that in the early stage, there was some significant impact on physical and social functions as well as in the symptom scales, such as fatigue and appetite loss. The results suggest that an assessment of physical and psychological issues is clinically important among those patients with gastric cancer so that patients can be referred for care to relieve problems of fatigue, appetite loss, diarrhea and financial difficulties.

As for patients with esophageal cancer, we found that the advanced group in general suffered from the most serious symptoms compared to the other groups. Dysphagia and trouble with coughing were two major early symptoms. Similarly, Seo-in Ha et al. found that dysphagia was one of the most common symptoms affecting the HR-QoL of patients with esophageal cancer [34]. Unlike a previous study, we found that as the disease worsened, HR-QoL areas of dry mouth and trouble with taste remained constant (i.e., no significant difference) [35]. We also found that the most severe symptom was reflux, which agrees with a previous study on HR-QoL in patients after esophageal cancer surgery [36]. Since there is an association between reflux and sleep problems, which may further lead to a series of other problems, useful measures should be taken to relieve this symptom [37]. It would be useful for health care providers to provide dietary guidance and counselling services for patients with esophageal disease to relieve their problems of reflux and insomnia.

As for patients with gastric cancer, the precancerous group and early stage group both reported better HR-QoL scores compared with the advanced stage group. This finding further confirms that people with advanced cancer had the worst HR-QoL. It is interesting to note that for most symptom scales, the HRQoL began to get worse in the early stage of cancer. This is consistent with the work by Axon et al. who showed that early gastric cancer usually presented with long-standing dyspepsia [38]. Therefore, doctors should pay attention to those signal symptoms and undertake meticulous examination of the stomach to relieve symptoms. Body image was also one of the most severe problems. This can be explained as the digestive symptoms related to inadequate food intake could cause weight loss and further lead to body image disturbance among gastric cancer patients [39]. Health providers should therefore provide proper nutritional care to manage the symptoms better and improve patients' perception of body image.

We also explored the influencing factors of HR-QoL in patients with upper GI diseases. It was not surprising that pathological stage was the most important influencing factor of HR-QoL in this study. As the disease progresses, the HR-QoL of patients gradually deteriorates. The significant effect of residence area on the HR-QoL scores in certain scales, such as physical functioning, cognitive functioning, financial difficulties etc., could be explained by the different living conditions and economic levels between the two counties. We also found that gender was an important factor associated with constipation. Similarly, Forootan et al. found that constipation as a disorder in the gastrointestinal tract was more common in women than in men, and severe constipation was discovered more often in elderly women as compared to male patients [40,41]. Hu and Sellick reported that education level was a significant predictor of HRQoL and a higher education level was related to a higher HR-QoL among patients with GI cancer [42]. Similarly, our results showed that higher symptom scores were associated with a lower education level. Our results demonstrated a significant association between marital status and patients' cognition and insomnia, because poor marital status was associated with a higher level of psychological distress, which is detrimental to one's health [43]. Overall, it is needed to provide more supports for those elderly patients with lower education levels and poorer health states.

Results from the EORTC QLQ-OES18 module revealed that the pathological stage had greater effects on symptom scales, and other factors had little effects on those scales. In this regard, Yu-Ling Chang showed that advanced tumor stage had significant deleterious effects on several aspects of HR-QoL, such as eating difficulties, and there were no associations between any factors (age, gender and body weight loss) and esophageal-specific aspects of HR-QoL [35]. Therefore, it is important to carry out early diagnosis and treatment program and to conduct follow-up care since those patients with advanced cancer usually have worse HR-QoL.

Results from the EORTC QLQ-STO22 module showed that age, education level and pathological stage were major influencing factors of HR-QoL scores. A previous study has shown that stomach-specific symptoms (pain, eating restrictions, and anxiety) lessened with increasing age [44]. We also found that the age of the patients significantly influenced body image and hair loss. In addition, education level had an impact on symptoms among patients with gastric diseases that mainly involved pain, eating difficulties, dry mouth and body image. Similarly, Lin Chenxi et al. found that educational background had a positive correlation with HR-QoL among patients with gastric cancer [45]. Therefore, better health educations should be provided for those patients in rural areas to guide them to choose healthy lifestyles and thus improve their HR-QoL.

\section{Limitations}

Although this study provides valuable information for improving the HR-QoL of patients with upper GI diseases, there are still several limitations. Firstly, in terms of the influencing factors, the effect of surgery-related factors (e.g. type of surgical technique, adjuvant therapy, postoperative complications etc.) on HR-QoL, which have been found to be main factors that influence HR-QoL in previous studies, were not included in the questionnaire. Limited by data availability, we didn't include surgery-related factors, so our findings can not fully reflect the reasons affecting the HR-QoL of patients. Secondly, because the sample of this study involved only rural patients in two counties in Shandong province in China, the results could only reflect the HR-QoL of patients with upper GI diseases in high-risk rural areas. Based on this study, investigations with a larger sample size should be conducted to evaluate the HR-QoL for patients at different pathological stages of upper GI diseases in the future. Thirdly, as a cross-sectional study, it was impossible to make an analysis of repeated measurements of HR-QoL over time. However, this study still provides useful basic information for a further study. In addition, it was also a

Page $10 / 13$ 
limitation that we didn't analyze the relative importance of the significant factors against pathological stage due to the sample size. In the future, it is necessary to conduct a long-term follow-up investigation to test the above findings.

\section{Conclusion}

The HR-QoL of patients with advanced upper GI cancer was worst in most scales compared with patients with precancerous lesions and patients in the early stage group. However, we still need to pay attention to the early symptoms of upper GI diseases and take some useful measures to relieve the most severe symptoms (i.e. reflux for those with esophageal diseases and body image for those with gastric diseases). In addition, patients with upper GI diseases persistently have difficulties in social and financial aspects. As for influencing factors, residence area, age, education level and pathological stage were the main predictors of HR-QoL scores. These findings provide valuable information for health care providers in China so that they may take appropriate measures to manage the symptoms of patients with upper GI diseases, and thereby improving their HR-QoL.

\section{Declarations}

Ethics approval and consent to participate: This study has been approved by the Ethics Committees of the School of Public Health of Shandong University (No. 20140201). Written informed consent was obtained from all individual participants included in the study before data collection.

Consent for publication: Not applicable.

Availability of data and materials: Please contact author for data requests.

Competing interests: The authors declare that they have no conflicts of interest to disclose.

Funding: There was no funding of this study.

Authors' contributions: Tingting Qin: Conceptualization, methodology, formal analysis, writing-original draft, and writing-review and editing. Yuchen Shao: Conceptualization, methodology, and data curation. Nan Zhang: Conceptualization, Investigation and project administration. Jialin Wang: Supervision and project administration. Nengliang Yao: Conceptualization, and writing-review and editing. Deli Zhao: Project administration and resources. Weidong Liu: Project administration and resources. Roger. T. Anderson: Supervision, and writing-review and editing. Xiaojie Sun: Conceptualization, methodology, investigation, resources, supervision, project administration, and writing-review and editing.

Acknowledgements: The authors thank all the local participants for their support and hard work in launching and implementing this ongoing study.

\section{References}

1. Bray F, Ferlay J, Soerjomataram I, Siegel RL, Torre LA, Jemal A. Global cancer statistics 2018: GLOBOCAN estimates of incidence and mortality worldwide for 36 cancers in 185 countries. CA Cancer J Clin. 2018;68(6):394-424. doi:10.3322/caac.21492.

2. Feng R-M, Zong Y-N, Cao S-M, Xu R-H. Current cancer situation in China: good or bad news from the 2018 Global Cancer Statistics? Cancer communications. (London England). 2019;39(1):22-2. doi:10.1186/s40880-019-0368-6.

3. Wu C, Li M, Meng H, Liu Y, Niu W, Zhou Y, et al. Analysis of status and countermeasures of cancer incidence and mortality in China. Sci China Life Sci. 2019;62(5):640-7. doi:10.1007/s11427-018-9461-5.

4. Chen W, Sun K, Zheng R, Zeng H, Zhang S, Xia C, et al. Cancer incidence and mortality in China, 2014. Chin J Cancer Res. 2018;30(1):1-12. doi:10.21147/j.issn.1000-9604.2018.01.01.

5. You WC, Blot WJ, Li JY, Chang YS, Jin ML, Kneller R, et al. Precancerous gastric lesions in a population at high risk of stomach cancer. Cancer Res. 1993;53(6):1317-21.

6. Lage J, Uedo N, Dinis-Ribeiro M, Yao K. Surveillance of patients with gastric precancerous conditions. Best Pract Res Clin Gastroenterol. 2016;30(6):913-22. doi:10.1016/j.bpg.2016.09.004.

7. Omidvari AH, Meester RG, Lansdorp-Vogelaar I. Cost effectiveness of surveillance for GI cancers. Best Pract Res Clin Gastroenterol. 2016;30(6):879-91. doi:10.1016/j.bpg.2016.09.001.

8. Sitlinger A, Zafar SY. Health-Related Quality of Life: The Impact on Morbidity and Mortality. Surg Oncol Clin N Am. 2018;27(4):675-84. doi:10.1016/j.soc.2018.05.008.

9. Dorcaratto D, et al. ). Quality of Life of Patients with Cancer of the Oesophagus and Stomach. Cir Esp. 2011;89(10):635-44.

10. Detmar SB, Aaronson NK, Wever LD, Muller M, Schornagel JH. How are you feeling? Who wants to know? Patients' and oncologists' preferences for discussing health-related quality-of-life issues. J Clin Oncol. 2000;18(18):3295-301.

11. Blazeby JM, Vickery CW. Quality of life in patients with cancers of the upper gastrointestinal tract. Expert Rev Anticancer Ther. 2001;1(2):269-76.

12. Hanna L, Huggins CE, Furness K, Silvers MA, Savva J, Frawley H, et al. Effect of early and intensive nutrition care, delivered via telephone or mobile application, on quality of life in people with upper gastrointestinal cancer: study protocol of a randomised controlled trial. BMC Cancer. 2018;18(1):707. doi:10.1186/s12885-018-4595-z.

13. Al-Batran SE, Van Cutsem E, Oh SC, Bodoky G, Shimada Y, Hironaka S, et al. Quality-of-life and performance status results from the phase III RAINBOW study of ramucirumab plus paclitaxel versus placebo plus paclitaxel in patients with previously treated gastric or gastroesophageal junction 
adenocarcinoma. Ann Oncol. 2016;27(4):673-9. doi:10.1093/annonc/mdv625.

14. Kauppila JH, Ringborg C, Johar A, Lagergren J, Lagergren P. Health-related quality of life after gastrectomy, esophagectomy, and combined esophagogastrectomy for gastroesophageal junction adenocarcinoma. Gastric Cancer. 2018;21(3):533-41. doi:10.1007/s10120-017-0761-2.

15. Safieddine N, Xu W, Quadri SM, Knox JJ, Hornby J, Sulman J, et al. Health-related quality of life in esophageal cancer: effect of neoadjuvant chemoradiotherapy followed by surgical intervention. J Thorac Cardiovasc Surg. 2009;137(1):36-42. doi:10.1016/j.jtcvs.2008.09.049.

16. Berglund A, Byström P, Johansson B, Nygren P, Frödin JE, Pedersen D, et al. An explorative randomised phase II study of sequential chemotherapy in advanced upper gastrointestinal cancer. Med Oncol. 2010;27(1):65-72. doi:10.1007/s12032-009-9173-4.

17. Blazeby JM, Farndon JR, Donovan J, Alderson D. A prospective longitudinal study examining the quality of life of patients with esophageal carcinoma. Cancer. 2000;88(8):1781-7.

18. Avery K, Hughes R, McNair A, Alderson D, Barham P, Blazeby J. Health-related quality of life and survival in the 2 years after surgery for gastric cancer. Eur J Surg Oncol. 2010;36(2):148-54. doi:10.1016/j.ejso.2009.09.008.

19. $10.1016 /$ S0140-6736(16)31956-0 Ju-Fang Shi ea. (2016). Quality-of-life and health utility scores for common cancers in China: a multicentre cross-sectional survey. The Lancet, 388(Supplement 1), S29, doi:https://doi.org/10.1016/S0140-6736(16)31956-0.

20. Areia M, Alves S, Brito D, Cadime AT, Carvalho R, Saraiva S, et al. Health-related quality of life and utilities in gastric premalignant conditions and malignant lesions: a multicentre study in a high prevalence country. J Gastrointestin Liver Dis. 2014;23(4):371-8. doi:10.15403/jgld.2014.1121.234.hrq.

21. Tian J, Chen ZC, Wu B, Meng X. Comparison of quality of life between urban and rural gastric cancer patients and analysis of influencing factors. World J Gastroenterol. 2004;10(20):2940-3.

22. Tian J, Chen JS. Nutritional status and quality of life of the gastric cancer patients in Changle County of China. World J Gastroenterol. 2005;11(11):1582-6.

23. Wang H, Pan Y, Guo C, Li F, Xu R, Liu M, et al. Health-related quality of life among rural residents aged 45-69 years in Hua County, Henan Province, China: Results of ESECC Trial for esophageal cancer screening with endoscopy. Chin J Cancer Res. 2018;30(2):240-53. doi:10.21147/j.issn.10009604.2018.02.07.

24. Conroy T, Marchal F, Blazeby JM. Quality of life in patients with oesophageal and gastric cancer: an overview. Oncology. 2006;70(6):391-402. doi:10.1159/000099034.

25. Bosman FT, Hruban FC,RH, et al. (2010). WHO Classification of Tumours of the Digestive System ((ed 4)). International Agency on Research of Cancer, Lyon, France, 2010.

26. Aaronson NK, Ahmedzai S, Bergman B, Bullinger M, Cull A, Duez NJ, et al. The European Organization for Research and Treatment of Cancer QLQ-C30: a quality-of-life instrument for use in international clinical trials in oncology. J Natl Cancer Inst. 1993;85(5):365-76.

27. Blazeby JM, Conroy T, Bottomley A, Vickery C, Arraras J, Sezer O, et al. Clinical and psychometric validation of a questionnaire module, the EORTC QLQSTO 22, to assess quality of life in patients with gastric cancer. Eur J Cancer. 2004;40(15):2260-8.

28. Blazeby JM, Conroy T, Hammerlid E, Fayers P, Sezer O, Koller M, et al. Clinical and psychometric validation of an EORTC questionnaire module, the EORTC QLQ-OES18, to assess quality of life in patients with oesophageal cancer. Eur J Cancer. 2003;39(10):1384-94.

29. Zhao H, Kanda K. Translation and validation of the standard Chinese version of the EORTC QLQ-C30. Qual Life Res. 2000;9(2):129-37.

30. Dai Z, Lang W, Yang H, Tian J, Sun W, Pekbay B, et al. Validation of EORTC QLQ-OES18 for Chinese patients with esophageal cancer. Dis Esophagus. 2017;30(10):1-7. doi:10.1093/dote/dox046.

31. al. MQe. Development and Evaluation on Chinese Version of Measurement Instrument of Quality of Life for Patients with Stomach Cancer: EORTC QLQST022. Journal of Kunming Medical University. 2012;33(10):34-8.

32. Wen Y, Pan X-F, Huang W-Z, Zhao Z-M, Wei W-Q, Chen F, et al. Quality of Life for Patients with Esophageal/Gastric Cardia Precursor Lesions or Cancer: A One-year Prospective Study. Asian Pac J Cancer Prev. 2015;16(1):45-51. doi:10.7314/apjcp.2015.16.1.45.

33. Tian, Zhi, et al. Assessment on quality of life for esophageal cancer in different pathological stage. Modern Preventive Medicine. 2010;37(22):4203210.

34. Ha S-i, Kim K, Kim J-s. The influence of symptoms on quality of life among patients who have undergone oesophageal cancer surgery. European Journal of Oncology Nursing. 2016;24:13-9. doi:https://doi.org/10.1016/j.ejon.2016.08.002.

35. Chang YL, Tsai YF, Wu YC, Hsieh MJ. Factors relating to quality of life after esophagectomy for cancer patients in Taiwan. Cancer Nurs, 37(1), 4-13, doi:10.1097/NCC.0b013e318277dc53.

36. Schandl A, Lagergren J, Johar A, Lagergren P. Health-related quality of life 10 years after oesophageal cancer surgery. Eur J Cancer. 2016;69:43-50. doi:https://doi.org/10.1016/j.ejca.2016.09.032.

37. Jansson C, Nordenstedt H, Wallander MA, Johansson S, Johnsen R, Hveem K, et al. A Population-Based Study Showing an Association Between Gastroesophageal Reflux Disease and Sleep Problems. Clin Gastroenterol Hepatol. 2009;7(9):960-5. doi:https://doi.org/10.1016/j.cgh.2009.03.007.

38. Axon A. Symptoms and diagnosis of gastric cancer at early curable stage. Best Practice Research Clinical Gastroenterology. 2006;20(4):697-708. doi:https://doi.org/10.1016/j.bpg.2006.03.015.

39. Kim AR, Cho J, Hsu YJ, Choi MG, Noh JH, Sohn TS, et al. Changes of quality of life in gastric cancer patients after curative resection: a longitudinal cohort study in Korea. Ann Surg. 2012;256(6):1008-13. doi:10.1097/SLA.0b013e31827661c9.

40. Forootan M, Bagheri N, Darvishi M. Chronic constipation: A review of literature. Medicine. 2018;97(20):e10631. doi:10.1097/md.0000000000010631. 
41. Vazquez Roque M, Bouras EP. Epidemiology and management of chronic constipation in elderly patients. Clin Interv Aging. 2015;10:919-30. doi:10.2147/CIA.S54304.

42. Yan H, Sellick K. Quality of life of Chinese patients newly diagnosed with gastrointestinal cancer: a longitudinal study. Int J Nurs Stud. 2004;41(3):30919. doi:https://doi.org/10.1016/j.ijnurstu.2003.10.004.

43. Ren XS. Marital status and quality of relationships: the impact on health perception. Soc Sci Med. 1997;44(2):241-9.

44. Bae JM, Kim S, Kim YW, Ryu KW, Lee JH, Noh JH, et al. Health-related quality of life among disease-free stomach cancer survivors in Korea. Qual Life Res. 2006;15(10):1587-96.

45. Lin Chen-xi, et al. The quality of life and its influential factors among discharged patients with gastric cancer in rural area. Wuwei Gansu Journal of Capital Medical University. 2011;32(5):661-6. 